\title{
GITR interacts with the pro-apoptotic protein Siva and induces apoptosis
}

\author{
Cell Death and Differentiation (2002) 9, 1382-1384. doi:10.1038/sj.cdd.4401140
}

\section{Dear Editor,}

We have previously identified and characterised a murine member of the tumour necrosis factor receptor superfamily (TNFRSF), named GITR (TNFR18). ${ }^{1-4}$ Most TNFRSF members modulate cell death and induce or counter apoptosis depending on activation of a specific transduction pathway and we showed GITR counters T cell apoptosis. This effect correlates in part with GITR/TRAF-2 binding and consequent NF- $\kappa$ B activation. ${ }^{5,6}$ The GITR cytoplasmic region shows a striking homology with the cytoplasmic region of other TNFRSF members such as CD40 (TNFRSF5), OX40 (TNFRSF4), 4-1BB (TNFRSF9) and CD27 (TNFRSF7) (Figure 1A). ${ }^{2}$ CD27 stimulation is reported to either protect from, or induce apoptosis, ${ }^{7}$ and, in fact, CD27 binding to Siva, a death-domain containing cytoplasmic molecule that is involved in the apoptotic response to virus infection and oxidative stress, ${ }^{7-9}$ induces apoptosis activation. On the basis of CD27 and GITR homology at the cytoplasmic domain (Figure 1A) we hypothesised that murine GITR (mGITR) could bind murine Siva (mSiva).

To verify whether mGITR and mOX40 bind mSiva, we in vitro translated their cytoplasmic domains together with the mCD27 domain as positive control. The $\left[{ }^{35} \mathrm{~S}\right]$ translated products were challenged with the fusion protein GSTmSiva (glutathione S-transferase-GST-fused to mSiva and bound to a sepharose resin) and extensively washed. A GST protein was used as negative control. Figure 1B shows that mGITR, mOX40 and mCD27 bound mSiva. A symmetric experiment using GST-mGITR (cytoplasmic region) and in vitro translated mSiva, confirmed mGITR/ mSiva binding (Figure 1C).

To test whether mGITR binds mSiva in vivo, we performed co-immunoprecipitation studies with tagged proteins (mGITR-myc and mSiva-Xpress), overexpressed in Cos7 cells. mSiva-Xpress or mGITR-myc were coimmunoprecipitated with the anti-myc or anti-Xpress antibody $(\mathrm{Ab})$ respectively and revealed by Western blot analysis. Results in Figure 1D indicate that mGITR binds mSiva in vivo. To check any aspecificities due to immunoprecipitating antibodies, lysates from GITR-myc and Siva-Xpress single transfection, immunoprecipitated with anti-Xpress or anti-myc Ab respectively, were used as negative controls. We also evaluated the level of mGITRmyc expression in comparison to that of mGITR in DEXtreated hybridoma T-cells ${ }^{1}$ or activated T-lymphocytes ${ }^{4}$ by semiquantitative PCR or cytofluorimetric experiments, respectively. Results indicate that similar levels of expres- sion are detected in transfected Cos7 cells and either activated or DEX treated T-cells (data not shown).

To identify the mGITR motif responsible for mSiva binding, we assessed the binding efficacy of 10 in vitro translated mutants of mGITR cytoplasmic domain (Figure $1 \mathrm{E})$. The percentage of the product bound to the fusion protein GST-mSiva compared to the input was quantitated upon SDS-PAGE separation. Figure $1 F$ and $G$ show that mGITR deleted in its C-terminal domain (GITR $\Delta 30$, mutant 1) did not bind mSiva, suggesting the $30 \mathrm{C}$-terminal amino acidic residues are necessary for mSiva binding. We then investigated the binding ability of several deleted mutants lacking 7-10 amino acidic residues in the C-terminal region (Figure 1E) and observed that GITR $\Delta \mathrm{c}$ (mutant 4) did not bind mSiva; GITR $\Delta a$ (mutant 2), GITR $\Delta \mathrm{b}$ (mutant 3) and GITR $\Delta d$ (mutant 5) bound mSiva less than GITR; and GITR $\Delta$ e (mutant 6 ) bound mSiva to a similar extent as GITR (Figure $1 \mathrm{~F}$ and $\mathrm{G}$ ). The lack of GITR $\Delta \mathrm{c}-\mathrm{mSiva}$ binding suggests that the amino acidic residues that are not present in GITR $\triangle \mathrm{c}$ (SFQFPEEE) are required for $\mathrm{mGITR} / \mathrm{mSiva}$ binding. The low binding degree of GITR $\Delta \mathrm{a}-\mathrm{mSiva}$ suggests that the amino acidic residues that are absent in GITR $\Delta a$, and N-flanking those missing in GITR $\Delta c$ (QLSAEDAC) play some role. Consequently, we prepared and studied specific mGITR protein mutants (Figure 1E) and found that GITR(S180A) (mutant 7), GITR(P190A) (mutant 9) and GITR(E191R,E192V,E193V) (mutant 10) did not bind mSiva, while GITR(E182R,C185G) (mutant 8) bound mSiva to a similar extent as mGITR (Figure $1 \mathrm{~F}$ and $\mathrm{G}$ ).

The lack of binding of GITR $\Delta \mathrm{c}$, mutant 7, mutant 9 and mutant 10 suggests that the GITR-Siva binding requires specific amino acidic domains. In particular, results indicate that the SFQFPEEE domain (position 186-193) is crucial for mSiva binding, with the PEEE sequence playing an important role. In human GITR (hGITR), the QFPEEE sequence is conserved, suggesting this sequence plays a functional role. Prasad et $a l^{7}$ and the present results demonstrate that mCD27, hCD27 as well as mOX40 bind Siva. Within these TNFRSF members a PIQE sequence is found in the region corresponding to the PEEE sequence of mGITR and hGITR (see Figure 1A). Thus, the core motif responsible for Siva binding might be $P(I / E)(Q / E) E$. The same motif is also present in human 4-1BB and a similar one (PQEE) is found in murine 4-1BB. In human and murine CD40 the PIQE motif is also present, though in different positions of the cytoplasmic domain. Therefore 
A 4-1BB h T---QEEDGCSCR--FP--EEE----EGGC4-1BB TH TGAOEFDACSCR-C-P--OEE----EGGGG CD27 h SC-PREEEG-STI---PI-QEDYRKPEPACS CD27 m SC-PREEEG-SAI---PI-QEDYRKFEPAFY CD40 h TAAPVOE---TLHGCQPVTQEDGK--ESRIS CD40 m TAA $\overline{P V Q E}---T L H G C Q P V T$ QEDGK--ESRIS GITR h ST----EDARSC--QFP--EEE-R-GE-R-S GITR h2 ST----EDARSC--OFP--EEE-R-GE-R-S GITR $m$ SA----EDACSF--QFP--EEE-R-GE--OT $0 X 40 \mathrm{~h}$------GGSFR--TPI-QEEQ--ADAHS I OX40 m -------GNSER--T

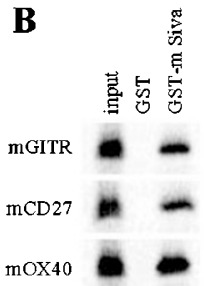

B

$\mathbf{E}$ GITR

(1) GITR $\Delta 30$

(2) GITR $\Delta a$

(3) GITR $\Delta$ b

(4) GITRAC

(5) GITRAd

(6) GITR $\Delta$ e

(7) GITR (S180A)

(8) GITR (E182R, C185G)

(9) GITR (P190A)

(10) GITR (E191R, E192V, E193V)

$160 \quad 170 \quad 180$

180

190

C

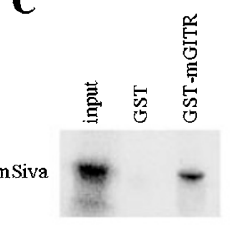

200

ITQ LRROHMCPRE TQPFAEVQL

STQRP BEERGEQTEE KCHLGRWPM MU IWQ LRRQHMCPRE TQPFAEVQLS A.................... E EEERGEQTEE KCHLGGRWPM MM

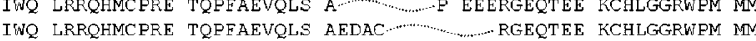
IWQ LRRQHMCPRE TQPFAEVQLS AEDACSFQFP....................... KCHLGGRWPM MM IWQ LRRQHMCPRE TQPFAEVQLS AEDACSFQFP EEERG ……….....HLGGRWPM MM IWQ LRRQHMCPRE TQPFAEVQLA AEDACSFQFP EEERGEQTEE KCHLGGRWM MM IWO LRROHMCPRE TOPFAEVOLS ARDAGSFOFP EEERGEOTEE KCHLGGRWM MM UQ URRQHMCPRE TQPEAEVQLS AEDACSTQTA EEERGEQTEE KCHLGGRWMM IWQ LRRQHMCPRE TQPFAEVQLS AEDACSFQFP RVVRGEQTEE KCHLGGRWPM MM

$\mathbf{H}$

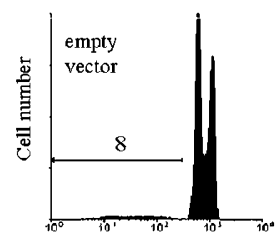

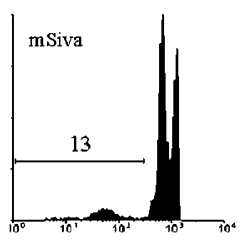

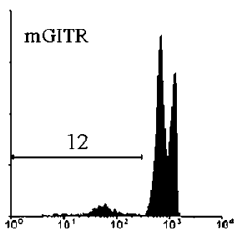

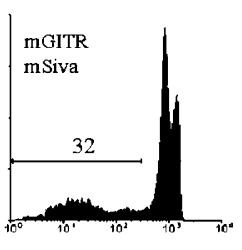

D anti-Xpress i.p. anti-myc i.p.

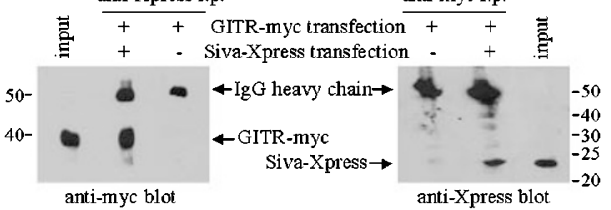

$\mathbf{F}$
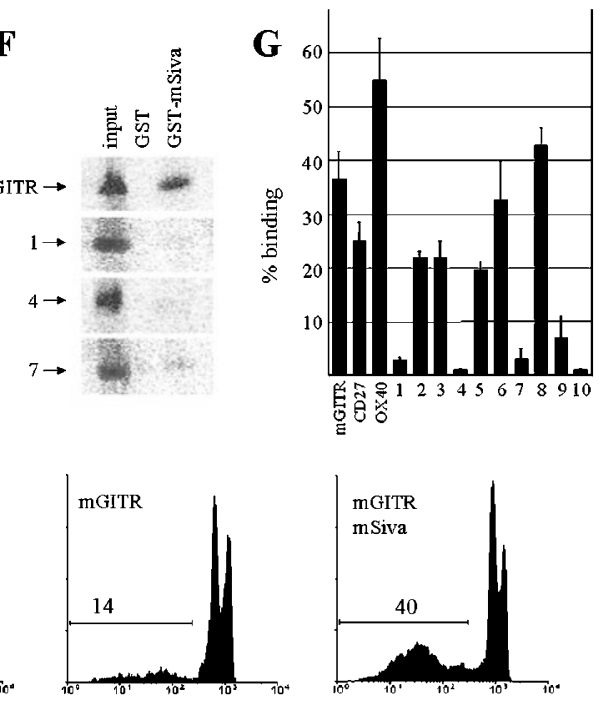

anti-GITR triggering

Figure 1 (A) Identity in a short sequence of the cytoplasmic domain among 4-1BB, CD27, CD40, GITR and OX-40 (murine, m; human, h). A human GITR splicing is also represented (h2). ${ }^{5,6}$ Identical amino acids or those with similar function $(\mathrm{R}, \mathrm{K}, \mathrm{H} ; \mathrm{D}, \mathrm{E} ; \mathrm{S}, \mathrm{T} ; \mathrm{I}, \mathrm{V} ; \mathrm{A}, \mathrm{G})$ in at least six chains are in bold characters. The conserved consensus sequence that may bind Siva is in bold characters, underscored and shaded. The CKII consensus sequence present in mGITR and hGITR is in bold characters and underscored. (B) The ${ }^{35} \mathrm{~S}$-labelled cytoplasmic domains of mGITR, mCD27 and mOX40 bind GST-mSiva fusion protein. The [ ${ }^{35}$ S]proteins before challenging with the fusion proteins are shown as input. No or insignificant binding was observed with the negative control (GST). (C) The ${ }^{35}$ S-labelled mSiva binds GST-mGITR fusion protein. The [ ${ }^{35}$ S]protein before challenging with the fusion protein is shown as input. No binding was observed with the negative control (GST). (D) On the left panel, the anti-Xpress Ab against mSiva-Xpress immunoprecipitates mGITR-myc (40 kDa) in lysates (mild Nonidet P-40 buffer) ${ }^{7}$ of Cos7 cells co-transfected with mSiva-Xpress and mGITR-myc. Western blot was performed by using anti-myc Ab. On the right panel, the anti-myc Ab against mGITR-myc immunoprecipitates mSiva-Xpress $(24 \mathrm{kDa})$ in lysates of Cos 7 cells co-transfected with mSiva-Xpress and mGITR-myc. Western blot was performed by using anti-Xpress Ab. Antibodies were precipitated by using the G-protein, lysates were subjected to a pre-clearing with G-protein and immunoprecipitates were washed five times (three with the lysis buffer and two with PBS). (E) mGITR cytoplasmic domain and its mutants as obtained by in vitro translation. Sixteen amino acids belonging to the GITR transmembrane domain were also in vitro translated (not shown). The three methionine residues at the C-terminal of the translated protein, do not belong to the cytoplasmic domain of GITR but were added to increase specific activity of ${ }^{35} \mathrm{~S}$-methionine labelled proteins. Numbers indicate the position of the amino acids in the mGITR mature protein and the asterisk indicates the CKII phosphorylation site. Amino acidic residues mutated in the last four mutants are underscored. (F) The ${ }^{35}$ S-labelled cytoplasmic domain of mGITR binds GST-Siva fusion protein, while GITR $\Delta 30$ (mutant 1), GITR $\Delta \mathrm{c}$ (mutant 4 ) and GITR(S180A) (mutant 7) do not. For details see Figure 1B. (G) Quantitation (by Instant Imager-Packard) of in vitro binding of mGITR, mCD27, mOX40, and mGITR mutants to mSiva. C.p.m. values of GST bound ${ }^{35}$ S]protein (negative control) were subtracted to that of GST-Siva bound [ ${ }^{35}$ S]protein and compared to the c.p.m. of the respective input. GITR mutants are indicated by numbers whose meaning is specified in Figure 1E. (H) Increased apoptosis in cells co-transfected with mGITR-myc and mSiva-Xpress. Cos7 cells were transfected with Fugene (Invitrogen) with the empty vector alone, mSiva-Xpress together with the empty vector, mGITR-myc together with the empty vector, or mGITR-myc together with mSiva-Xpress. Twenty-four hours later, cells were stimulated or not with the anti-GITR antibody and the percentage of apoptotic cells was evaluated by cytofluorimetric analysis of PI stained cells ${ }^{13} 24 \mathrm{~h}$ later. One of three similar experiments is shown

each of the TNFRSF members reported in Figure $1 \mathrm{~A}$ might bind Siva.

In mGITR, the QLSAEDAC sequence (position 178185) also plays a role in mSiva binding. Inside the sequence, substitution of E182 and C185 did not modify binding, while S180, a potential Casein Kinase II (CKII) phosphorylation site $\left(S X_{2} E / D\right),{ }^{10}$ was necessary for mSiva binding (see mutant 7 in Figure $1 F$ and $G$ ). In hGITR, the consensus for the CKII phosphorylation site is conserved (STED vs SAED of mGITR), providing further evidence that the Ser/Thr phosphorylation site potentially has a functional role. As no conserved CKII phosphorylation sites are found in the cytoplasmic regions of $4-1 B B, C D 27, C D 40$ and
OX40, this phosphorylation site appears to be peculiar to GITR.

To verify whether the $\mathrm{mGITR} / \mathrm{mSiva}$ association has a functional role, mGITR and mSiva were co-transfected in Cos7 cells. Results of a representative experiment in Figure $1 \mathrm{H}$ show that transfection of either mGITR-myc or mSivaXpress did not induce apoptosis, while co-transfection did. As overexpression of the TNFRSF members is generally accepted to promote low levels of activation, we can assume cotransfection mimics a sort of mGITR triggering. In fact, a potentiated similar effect was obtained by triggering GITR with an anti-mGITR antibody directed against the extracellular region of mGITR (Figure $1 \mathrm{H}$ ). 
These results further suggest that the GITR-Siva binding shown in Figure $1 \mathrm{~B}, \mathrm{C}$ and $\mathrm{D}$ represents an in vivo interaction.

In conclusion, these data demonstrate that mGITR binds and activates mSiva, which leads to cell death. In mGITR, PEEE (position 190-193) is the crucial motif responsible for mSiva binding, like the PIQE sequence in CD27 and OX40, which is probably responsible for mSiva binding to the cytoplasmic domain of these receptors.

Other TNFRSF members, including CD27, have been reported to regulate T-lymphocyte development as they can either induce or counter apoptosis ${ }^{11,12}$ and, indeed, CD27mediated induction of apoptosis is mediated via Siva binding. $^{7}$ Consequently, mGITR binding with the proapoptotic protein mSiva is in line with its potential role in the control of $\mathrm{T}$ cell death and proliferation. ${ }^{1-4}$

\section{Acknowledgements}

This work was supported by Associazione Italiana Ricerca sul Cancro (AIRC) Milan, Italy.

\author{
S Spinicelli ${ }^{1,2}$, G Nocentini ${ }^{1,2}$, S Ronchetti ${ }^{1}$, LT Krausz ${ }^{1}$, \\ $R$ Bianchini $^{1}$ and $C$ Riccardi ${ }^{*}, 1$
}

${ }^{1}$ Department of Clinical and Experimental Medicine, Section of Pharmacology, Perugia University Medical School, 06100 Perugia, Italy ${ }^{2}$ The first two authors equally contributed to this work.

${ }^{*}$ Corresponding author: Carlo Riccardi, Department of Clinical and Experimental Medicine, Section of Pharmacology, Perugia University Medical School, 06100 Perugia, Italy. Tel: +390755857468;

Fax:+390755857405; E-mail: riccardi@unipg.it

1. Nocentini $G$ et al. (1997) Proc. Natl. Acad. Sci. USA 94: 6216-6221

2. Nocentini $G$ et al. (2000) DNA Cell Biol. 19(4): 207-219

3. Nocentini $G$ et al. (2000) Cell Death Differ. 7: 408-410

4. Ronchetti S et al. (2002) Blood 100(1): 350-352

5. Kwon B et al. (1999) J. Biol. Chem. 274(10): 6056-6061

6. Gurney AL et al. (1999) Current Biol. 9(4): 215-218

7. Prasad KV et al. (1997) Proc. Natl. Acad. Sci. USA 94: 6346-6351

8. Henke A et al. (2000) J. Virol. 74: 4284-4290

9. Cao C et al. (2001) J. Biol. Chem. 276: 11465-11468

10. Pinna LA (1990) Biochim. Biophys. Acta. 1054: 267-284

11. Wallach D et al. (1998) Curr. Opin. Immunol. 10: 279-288

12. Grammer AC and Lipsky PE (2000) Adv. Immunol. 76: 161-178

13. Migliorati $G$ et al. (1993) Blood 81: 1352-1358 